\title{
Comparative Analysis of AM and MZ Modulator in 2×100 Gbps Based WDM Optical Network
}

\author{
HimaniRathore $^{1}$, Ankita R. Mowar ${ }^{2}$, Dr.SoniChanglani ${ }^{3}$ \\ M.Tech Scholar, ECE Department, LNCTS, Bhopal MP, India ${ }^{1}$ \\ A.P., ECE Department, LNCTS, Bhopal MP, India ${ }^{2}$ HOD,ECE Department,LNCTS,Bhopal MP,India ${ }^{3}$
}

\begin{abstract}
Multiplexing is widely employed due to its capability to increase transmission capacity and to reduce system costs. The system features multiplexing of the basebands in electrical domain as well as multiplexing in optical domain (WDM). The design is proposed for 10- users, each one assigned a different RZ duty cycle and with a data rate of 20 Gbps. In this work, optical wavelength Division Multiplexing is done on wavelengths $1550 \mathrm{~nm}$ and $1552 \mathrm{~nm}$. There are two modulation schemes AM and MZ are compared for $2 \times 100$ Gbps Based WDM Optical Network. The AM shows better performance as compared to MZ modulation scheme, AM transmitted successfully upto $78 \mathrm{~km}$ where as MZ up to $75 \mathrm{~km}$.
\end{abstract}

Keywords: WDM, BER, AM, Mach-Zehnder

\section{INTRODUCTION}

Multiplexing is one of the fundamental necessities in In wavelength division Multiplexing (WDM) system the today's digital communications. Multiplexing allows Mach-Zehnder (MZ) modulator plays an important role multiple users (or data inputs) to share the bandwidth of and a single modulator are used to modulate the transmission medium. In existing systems, the medium severalimmobile wavelengths or single variable is normally shared based on time slot (TDM), carrier wavelength[1]. Both of low frequency and high frequency frequency (FDM) or spectrum coding (CDM). The goals Components are collected by Amplitude Modulated signal. of all multiplexing techniques are to support as many users at as high speed and at the lowest cost possible [3].

The process of mixing the information to an optical and electrical signal is called modulation and in this process use to a device to modulate the information is called modulators. The more importantkinds of modulators in optical communication are Mach-Zehnder (MZ) modulator, Amplitude Modulator (AM), Phase Modulator (PM), Frequency Modulator (FM) and Electro absorption Modulator[4].The basic diagram of modulator is shown in figure.1

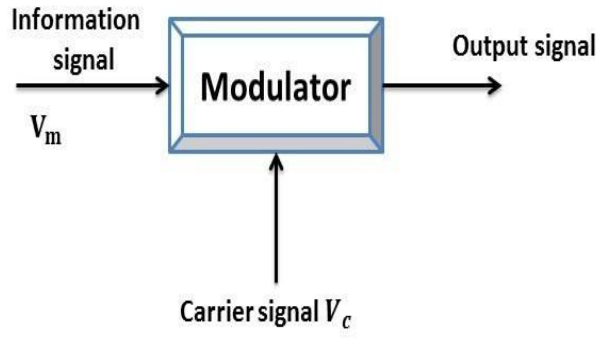

Figure.1 Concept of Modulator

In optical fibre communication the number of optical modulators requirements by used in large capacity photonic networks. Radio over Fibre (RoF) systems to producemillimeter-waves by using the external electrooptic modulator is named Mach-Zehnder modulator.
The low Frequency signals are generate the envelope of the modulating signal. The Acoustic signal is baptized low frequency signal and the AM radio station would be the transmitting the high frequency signal. In phase modulation the carriers phasevariationallowing to the message signal.

If carrier frequency is modificationallowing to the message signal that is termed FM and the bandwidth of $\mathrm{AM}$ is a smaller amount as associate to bandwidth of FM. In FM system the signal's information bandwidth is less than the signal's transmission bandwidth.

Due to the presence of an electric field the transformation of the optical absorption Coefficient in materials represents by the electro absorption (EA) consequence. The materials of EAMs are working the optical wavelengths at 1.3 to $1.6 \mu \mathrm{m}$.

\section{SIMULATION SETUP}

Figure. 2 shows simulation setup of 10-user WDM Optical Network, the signals are generated by Return-to-zero pulse generators with different duty cycle and electrically multiplexed by adders. 


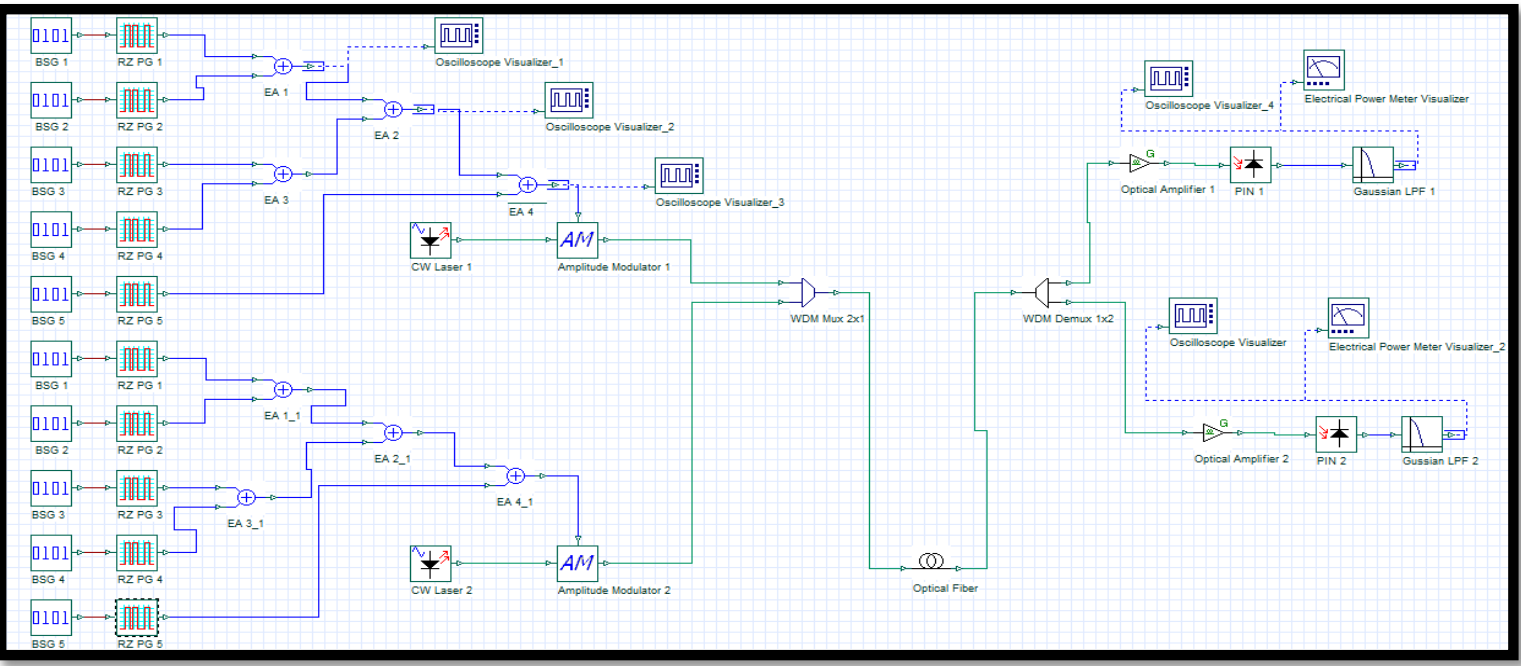

Figure.2 Simulation setup for 2×100 GbpsWDM Optical Network

\section{III.RESULTS}

Fig. 3 shows BER comparison of AM and MZ Modulator for 2×100 Gbps Based WDM Optical Network. The AM shows better performance as compared to MZ modulation scheme, AM transmitted successfully up to $78 \mathrm{~km}$ where as MZ up to $75 \mathrm{~km}$.
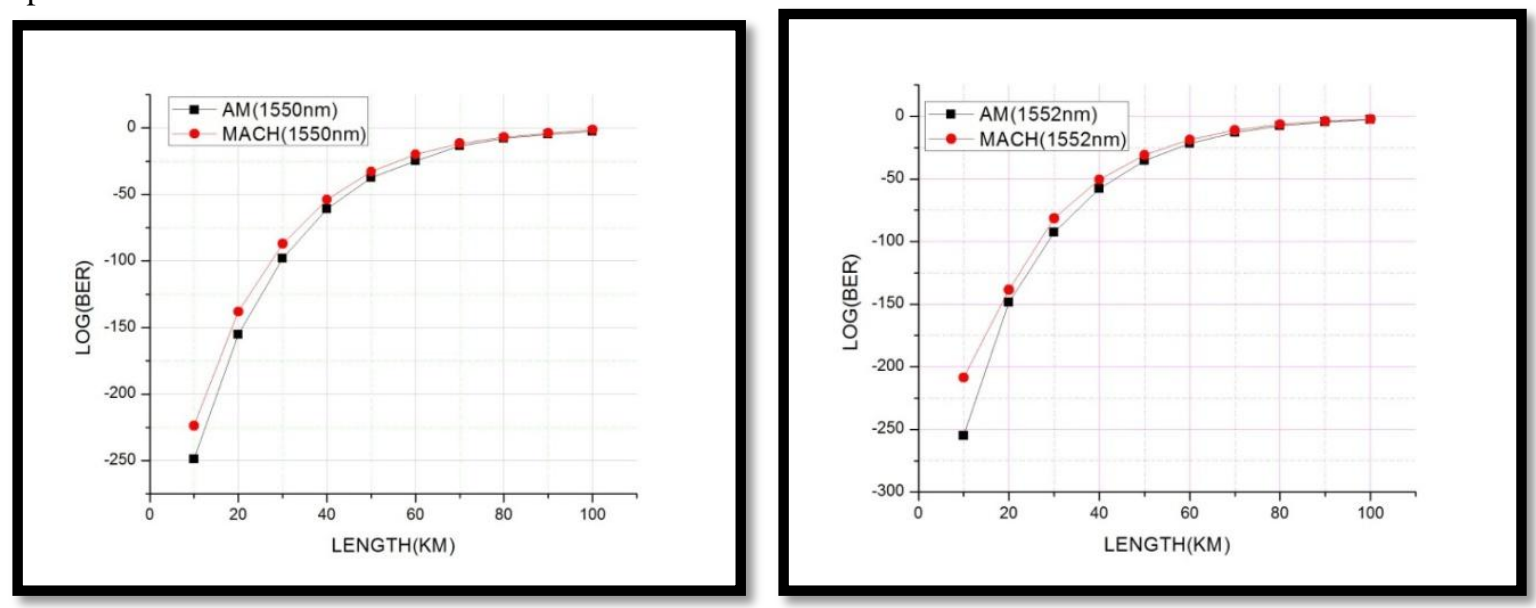

Figure.3 Log BER Vs Length for AM and MZ modulators

\section{IV.CONCLUSION}

The $2 \times 100$ Gbps Based WDM Optical Network is successfully designed. A technique of AM shows better performance as compared to MZ modulation scheme and AM transmitted suceessffullyupto $78 \mathrm{~km}$ where as $\mathrm{MZ}$ up to $75 \mathrm{~km}$ using SSMF.

\section{REFERENCES}

[1] HimaniRathore, AnkitaR. Mowar and Dr.SoniChanglani" Design the $2 \times 100$ Gbps Based Hybrid WDM Optical Network" INTERNATIONAL JOURNAL OF INNOVATIVE RESEARCH IN ELECTRICAL, ELECTRONICS, INSTRUMENTATION AND CONTROL ENGINEERING Vol. 2, Issue x, Xxxxx 2014

[2] Avinash Singh, Gireesh G. Soni, AbhishekTraipathi and AnuragShrivastava "Design of $3 \times 60$ Gbps DCDM based WDM system" 2012 International Conference on Optical Engineering (ICOE) in IEEE 2012

[3] M. K. Abdullah, G. A. Mahdiraji, A. M. Mohammadi, M. Mokhtar and A. F. Abas "Duty Cycle Division Multiplexing (DCDM), A New Electrical Multiplexing Technique for High Speed Optical Communication Systems", Proceedings of IEEE 2008 6th National Conference on Telecommunication Technologies and IEEE 2008, 2nd Malaysia Conference on Photonics, 26-27 August 2008, Putrajaya, Malaysia
[4] J. H. Lee, S. Ohara, T. Nagashima, T. Hasegawa, N. Sugimoto, K.Igarashi, K. Katoh, and K. Kikuchi, "Clock recovery and demultiplexing of high-speed OTDM signal through combined use of bismuth oxide nonlinear fiber and erbium-doped bismuth oxide fiber," IEEE Photonics Technology Letters, vol. 17, no. 12, pp. 2658-2660, Dec. 2005.

[5] S. A. Aljunid, M. Ismail, A. R. Ramli, B. M. Ali, M. K. Abdullah, "A new family of opticalcode sequences for spectral-amplitudecoding optical CDMA systems", Photonics TechnologyLetters, Vol. 16 , Issue 10 , pp. 2383-2385, (2004) 